\section{Síndrome de regresión embrionaria testicular: conceptos clínicos y revisión de la bibliografía}

Morales-Aranda JJ, Vázquez-Niño CL, Gutiérrez-Rosales R, Aragón-Castro MA, Ruvalcaba-Oceguera GE

\begin{abstract}
Resumen
Los trastornos del desarrollo sexual tienen baja incidencia en la población pediátrica; sin embargo, su repercusión a mediano y largo plazo justifica su entendimiento. El síndrome de regresión embrionaria testicular es una alteración compleja, que frecuentemente pasa inadvertida en el diagnóstico médico; se caracteriza por la exposición embrionaria al estímulo de la testosterona, pero en algún proceso del desarrollo sucede una regresión gonadal, que resulta o expresa un fenotipo masculino en grado variable. El diagnóstico se establece con la detección del cariotipo 46, XY, determinación de testosterona poscastración y concentraciones séricas elevadas de LH y FSH. La concentración sérica de hormona antimülleriana representa un marcador indirecto de coexistencia y función testicular; en pacientes con concentraciones normales o elevadas debe sospecharse un trastorno en la síntesis androgénica y, de esta manera, se excluye el diagnóstico de síndrome de regresión embrionaria testicular. El tratamiento aún se discute, pues debe individualizarse según el grado de virilización fenotípica, pero siempre debe ser multidisciplinario. Es importante ofrecer apoyo psicológico para el paciente y sus padres.
\end{abstract}

PALABRAS CLAVE: regresión embrionaria testicular, trastornos del desarrollo sexual, uropediatría, intersexo.

Rev Mex Urol. 2017 may;77(3):238-243.

\section{Embryonic testicular regression syndrome: clinical concepts and a literature review}

Morales-Aranda JJ, Vázquez-Niño CL, Gutiérrez-Rosales R, Aragón-Castro MA, Ruvalcaba-Oceguera GE

\section{Abstract}

SEXUAL development disorders have a low incidence in the pediatric population, but given their intermediate and long-term impact on the life of the patients, they need to be understood. Embryonic testicular
Departamento de Urología, Instituto de Seguridad Social del Estado de México y Municipios, Toluca, Estado de México, México

Recibido: diciembre 2016

Aceptado: marzo 2017

Correspondencia

Dr. Juan José Morales Aranda

drjuanjosemorales@gmail.com

Este artículo debe citarse como

Morales-Aranda JJ, Vázquez-Niño CL, GutiérrezRosales R, Aragón-Castro MA, Ruvalcaba-Oceguera GE. Síndrome de regresión embrionaria testicular: conceptos clínicos y revisión de la bibliografía. Rev Mex Urol. 2017 mayo;77(3):238-243. 
regression syndrome is a complex entity that is often unperceived in the medical diagnosis. It is characterized by embryonic exposure to testosterone stimulus, but at some point of development, gonadal regression occurs, resulting in or expressing varying degrees of the masculine phenotype. Diagnosis is made through the detection of the 46 , $\mathrm{XY}$ karyotype and the determination of castration levels of testosterone, as well as elevated serum luteinizing hormone and follicle-stimulating hormone levels. Serum concentration of the anti-Müllerian hormone is useful as an indirect marker of testicular presence and function. When levels are normal or increased, an androgenic synthesis disorder should be suspected, and embryonic testicular regression syndrome excluded. Management is controversial, because it is individualized according to the degree of phenotypic virilization, but it should always be multidisciplinary. Psychologic support is essential for both the patient and parents.

KEY WORDS: Embryonic testicular regression; Sexual development disorders; Pediatric urology; Intersex
Departamento de Urología, Instituto de Seguridad Social del Estado de México y Municipios, Toluca, Estado de México, México

Correspondence

Dr. Juan José Morales Aranda drjuanjosemorales@gmail.com

\section{ANTECEDENTES}

Los trastornos del desarrollo sexual derivan de una alteración en la cascada de eventos que determinan el sexo fetal y el desarrollo del sistema reproductivo. Su incidencia es de 1 en 4,000 niños. Estos trastornos pueden ser anatómicos, cromosómicos, genéticos, gonadales u hormonales, y pasar inadvertidos por falta de sospecha diagnóstica. ${ }^{1}$

El desarrollo sexual se inicia con la determinación del sexo de las gónadas embrionarias bipotenciales: los ovarios o los testículos, como un proceso derivado del complemento cromosómico. La diferenciación hacia ovario es un proceso predeterminado, a menos que un cromosoma $Y$ con una región SRY funcional permita estimular la transformación a testículo. Después de la determinación sexual masculina, las células de Sertoli y de Leydig son evidentes en la séptima y octava semanas de la vida gestacional, respectivamente. ${ }^{2}$

Antes de la determinación sexual gonadal, los embriones masculinos y femeninos tienen dos pares de ductos genitales: los müllerianos o paramesonéfricos, y los wolffianos o mesonéfricos, destinados a convertirse en estructuras genitales. ${ }^{2}$ En el feto denominado $46, \mathrm{XY}$, la secreción de la hormona antimülleriana por las células de Sertoli resulta en regresión del conducto mülleriano, mientras que la secreción de testosterona por las células de Leydig estabiliza los conductos wolffianos e induce su diferenciación hacia epidídimos, conductos deferentes y vesículas seminales. ${ }^{3}$ La diferenciación sexual de los genitales externos en los fetos 46, XY, se inicia con el desarrollo del falo durante la novena semana de gestación. La virilización de las estructuras masculinas depende de la coexistencia de dihidrotestosterona (DHT), 
decisiva para la fusión de los pliegues uretrales y la región labioescrotal. ${ }^{3,4}$

\section{Definición}

El síndrome de regresión embrionaria testicular se caracteriza por el cariotipo 46, XY, y anorquia, y el diagnóstico se establece cuando existe evidencia clara de la función testicular durante algún punto de la embriogénesis. ${ }^{5}$

Es una alteración en la que los testículos existieron, pero se atrofiaron y desaparecieron en etapas tempranas del desarrollo. La historia natural de dichos síndromes aún se desconoce, debido a la falta de estudios prospectivos, con cohortes de pacientes. ${ }^{6}$ La enfermedad puede ser unilateral o bilateral, con ausencia total o parcial de tejido testicular normal, incluso con genitales externos normales. ${ }^{6}$

\section{Etiología}

El origen del síndrome de regresión embrionaria testicular es incierto. El descenso incompleto de los testículos puede favorecer la torsión testicular fetal. También se ha propuesto algún tipo de traumatismo en el testículo intraescrotal durante la etapa prenatal, ${ }^{6}$ incluso micropérdida del cromosoma Y. El fenotipo depende de la etapa prenatal en la que ocurre el evento testicular catastrófico. ${ }^{7}$

\section{Cuadro clínico}

Desde el punto de vista clínico, este síndrome tiene diversas manifestaciones fenotípicas, según el grado de severidad: desde completamente femeninas hasta fenotipo masculino con microfalo y escroto vacío. ${ }^{3}$

El diagnóstico se establece con la detección del cariotipo 46, XY, determinación de testosterona poscastración y concentraciones séricas elevadas de $\mathrm{LH}$ y $\mathrm{FSH}^{3}$
En las formas más severas de síndrome de regresión embrionaria testicular es posible identificar agonadismo en el cariotipo 46, XY, con fenotipo femenino sin estructuras genitales internas. Esto resulta cuando el testículo produce hormona antimülleriana, pero desaparece al día 60 de la gestación, antes de generar andrógenos. En el síndrome de regresión embrionaria testicular verdadero, los pacientes pueden manifestar agonadismo con fenotipo $\mathrm{XY}$, desarrollo total de las estructuras wolffianas y escroto vacío, ausencia prostática y microfalo. Esto representa pérdida catastrófica testicular después del desarrollo genital total durante el primer trimestre de la gestación. ${ }^{4}$ Existen múltiples características clínicas que obligan a establecer un protocolo diagnóstico más profundo. Cuadro 1 y Figura 1.

\section{Estudios iniciales de laboratorio e imagen}

\section{Estudios genéticos}

Análisis cromosómico de rutina. Debe obtenerse inmediatamente en todos los pacientes con ambigüedad de genitales. La determinación del cariotipo es obligatoria. ${ }^{8}$ Ambos análisis deben efectuarse para detectar concomitancia de mosaicismo cromosómico en ciertas condiciones, pérdidas o duplicaciones (principalmente

Cuadro 1. Fenotipo de sospecha diagnóstica de trastornos del desarrollo sexual

Micropene con testículos no palpables bilateralmente.

- Micropene: Longitud peneana menor de $2.5 \mathrm{~cm}$ en infantes de término mediante tracción.

Clitoromegalia.

- Longitud del clítoris mayor de $9 \mathrm{~mm}$ o ancho mayor de $6 \mathrm{~mm}$.

Hipospadia penoescrotal o perineoescrotal en testículos no descendidos.

Fusión labial posterior.

- Índice anogenital, medido como la distancia entre el ano y la base del falo. Si es mayor de 0.5 representa un componente de diferenciación femenina y virilización. 
Morales-Aranda JJ y col. Síndrome de regresión embrionaria testicular

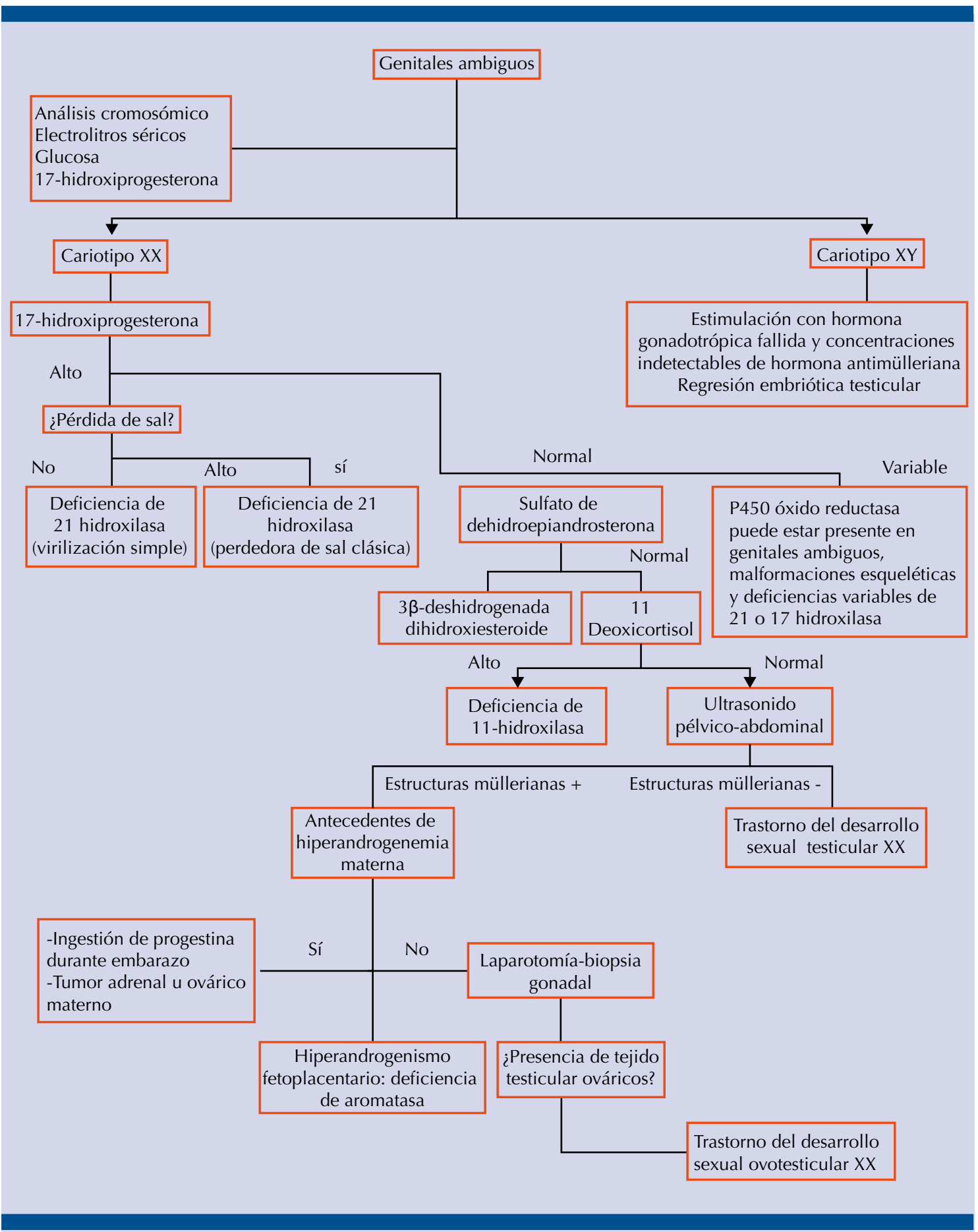

Figura 1. Algoritmo de genitales ambiguos. 
pérdida del SRY) que no son detectadas con los análisis cromosómicos de referencia. ${ }^{9}$

\section{Estudios hormonales (determinaciones)}

- 17-Hidroxiprogesterona. Se encuentra elevada en pacientes con deficiencia de 21-hidroxilasa.

- Electrolitos séricos. Deben solicitarse en todos los casos de ambigüedad genital, en busca de hiponatremia o hipercaliemia, que puede orientar hacia hiperplasia suprarrenal congénita, variante perdedora de sal. ${ }^{10}$

- Prueba de estimulación de hormona adrenocorticotrópica. Se realiza cuando la concentración de 17-hidroxiprogesterona no es concluyente o aún existe la posibilidad de hiperplasia suprarrenal congénita. ${ }^{10}$

- Concentraciones basales de hormona luteinizante y folículo estimulante.

- Concentración de testosterona. ${ }^{11}$

- Concentración de hormona antimülleriana. Es un indicador relativamente confiable de la coexistencia y función testicular. En caso de ser indetectable sugiere ausencia de tejido testicular; sin embargo, las concentraciones normales o elevadas señalan algún defecto en la síntesis de andrógenos o alteración en los receptores de andrógenos. ${ }^{12,13}$

- Prueba de estimulación de gonadotropina coriónica humana.

- Ultrasonografía pélvica-abdominal. Debe realizarse a todos los pacientes y, según los hallazgos, considerar la tomografía axial computada o resonancia magnética. ${ }^{12}$ Estos estudios permiten evaluar las características y localización de las gónadas.

\section{Tratamiento}

El tratamiento de pacientes con síndrome de regresión embrionaria testicular depende de las manifestaciones clínicas; sin embargo, el consenso sugiere un protocolo multidisciplinario y apoyo psicológico para el paciente y sus padres. Los fenotipos femeninos infantiles requieren suplementación estrogénica al momento de la pubertad esperada para el desarrollo de caracteres sexuales secundarios, incluso dilataciones vaginales o vaginoplastias. ${ }^{14,15}$

En fenotipos masculinos se requiere terapia androgénica a largo plazo, iniciando en la pubertad esperada. Los pacientes pueden beneficiarse de la colocación de prótesis testiculares. ${ }^{14,16}$

\section{Financiación}

No se recibiố patrocinio de ningún tipo para llevar a cabo este artículo.

\section{Conflicto de intereses}

Los autores declaran no tener ningún conflicto de intereses.

\section{REFERENCIAS}

1. Rey RA, Grinspan R. Normal male sexual differentiation and aetiology of disorders of sex development. Best Pract Res Clin Endocrinol Metabol. 2011;25:221-238.

2. Sajjad Y: Development of the genital ducts and external genitalia in the early human embryo. J Obstet Gynaecol Res 2010; 36:929.

3. Philibert $P$, Zenaty $D$, Lin $L$, et al. Mutational analysis of steroidogenic factor 1 (NR5a1) in 24 boys with bilateral anorchia: a French collaborative study. Hum Reprod 2007;22:3255-61.

4. Cohen-Kettenis P. Psychological long-term outcome in intersex conditions. Horm Res 2005;64(Suppl.):27.

5. Migeon CJ. Comments about the need for prenatal treatment of congenital adrenal hyperplasia due to 21-hydroxylase deficiency. J Clin Endocrinol Metab 1990;70:836-7. 
6. Sutcliffe JR, Wilson-Storey D, Smith NM. Ante-natal testicular torsion: only one cause of the testicular regression syndrome? J R Coll Surg Edinb 1996;41:99-101.

7. Calogero AE, Garofalo MR, Barone N, De Palma A, Vicari $E$, Romeo R, et al. Spontaneous regression over time of the germinal epithelium in a $Y$ chromosome-microdeleted patient: case report. Hum Reprod 2001;16:184-185.

8. Lambert SM, Vilain EJ, Kolon TF: A practical approach to ambiguous genitalia in the newborn period. Urol Clin N Am 2010;37:195 2.

9. Huhtaniemi IT. The role of mutations affecting gonadotrophin secretion and action in disorders of pubertal development. Best Pract Res Clin Endocrinol Metabol. 2002;16:123-138.

10. Thompson ST, Grillis MA, Wolkoff LH \& Katzin WE. Transverse testicular ectopia in a man with persistent Müllerian duct syndrome. Arch Pathol Lab Med. 1994;118:752-755.
11. Lee YS, Kirk JM, Stanhope RG, et al. Phenotypic variability in 17beta-hydroxysteroid dehydrogenase-3 deficiency and diagnostic pitfalls. Clin Endocrinol 2007;67:20-28.

12. Miller WL, Huang N, Agrawal V, Giacomini KM. Genetic variation in human $\mathrm{P} 450$ oxidoreductase. Mol Cell Endocrinol 2009;300:180-184.

13. Giordano SJ, Kaftory A, Steggles AW. A splicing mutation in the cytochrome b5 gene from a patient with congenital methemoglobinemia and pseudohermaphrodism. Human Genetics. 1994;93:568-570.

14. Hughes IA, Morel Y, McElreavey $K$, et al. Biological assessment of abnormal genitalia. J Pediatr Urol 2012;8:592-6.

15. Thyen $U$, Lanz K, Holterhus $P$, et al. Epidemiology and initial management of ambiguous genitalia at birth in Germany. Hormone Res 2006;66:195e203.

16. Parisi MA, Ramsdell LA, Burns MW, et al. A Gender Assessment Team: experience with 250 patients over a period of 25 years. Genet Med 2007;9:348.

\section{AVISO PARA LOS AUTORES}

Revista Mexicana de Urología tiene una nueva plataforma de gestión para envío de artículos: https://www.revisionporpares.com/index.php/RMUrol ahí podrá inscribirse a la base de datos administrada por el sistema Open Journal System (OJS) que ofrece las siguientes ventajas para los autores:

- Subir sus artículos directamente al sistema.

- Conocer, en cualquier momento, el estado de los artículos enviados, es decir, si ya fueron asignados a un revisor, aceptados con o sin cambios, o rechazados.

- Participar en el proceso editorial corrigiendo y modificando sus artículos hasta su aceptación final. 